\title{
PRODUCTIVIDAD: \\ Una propuesta desde la gestión del conocimiento
}

\begin{abstract}
RESUMEN
Los problemas de la organizaciones
están relacionados con las
deficiencias y dificultades para
realizar procesos efectivos de
monitoreo, seguimiento y control de
las acciones y actividades que
realiza la empresa para la
generación de productos y servicios
que respondan a las expectativas y
necesidades de clientes y mercados.
La productividad entendida como el
uso efectivo de las capacidades y
recursos de la organización en la
fabricación de productoso prestación
de servicios esta asociada al
conocimiento que las personas
tienen de las acciones y actividades
que se ejecutan en los diversos
procesos realizados. Esto implica
vincular los temas de gestión del
conocimiento, control total de
pérdidas y productividad.
\end{abstract}
\section{Palabras clave}

Gestión del conocimiento, control total de pérdidas, productividad, competitividad.
Por:

Nofal Nagles García*

\section{INTRODUCCIÓN}

\begin{abstract}
E
n el actual escenario empresarial altamente competitivo en que sólo sobreviven las organizaciones que evidencian una gran capacidad de adaptación, una elevada agilidad y flexibilidad para desarrollar acciones efectivas y generar rápidamente respuestas ingeniosas y creativas a las dificultades y desafíos que plantea el escenario competitivo.
\end{abstract}

En estas circunstancias es necesario desarrollar la capacidad creativa y la habilidad para generar soluciones novedosas e innovadoras a los problemas y dificultades, así como, a las demandas de los clientes. Una forma para lograr un buen desempeño en este escenario es utilizar el conocimiento para agregar valor en forma creativa en los productos y servicios que aseguren la sustentabilidad y sostenibilidad de las organizaciones en el tiempo y espacio.

\footnotetext{
* Ing.Industrial, especialista en Gerencia de Procesos de Calidad e Innovación, Master Dirección de Operaciones Calidad e Innovación, Doctorando en Ciencias Empresariales.
} 
Desde esta perspectiva se requiere comprender las capacidades, los recursos y los conocimientos que posee la organización y que utiliza en la fabricación de productos o la prestación de servicios que entrega a los clientes, consumidores y mercados. En consecuencia, entender qué sabe y cómo aplica este saber la empresa en sus procesos, acciones y actividades permite desarrollar ventajas competitivas sostenibles.

Para lograr la sostenibilidad la empresa necesita ser productiva y enfrentar de manera creativa y novedosa las pérdidas potenciales y esto se puede hacer desde la gestión del conocimiento.

La gestión del conocimiento se ha venido aplicando con éxito por parte de empresas de vanguardia en Estados Unidos y Europa, que disfrutan de los beneficios logrados. En Colombia, ha comenzado su aplicación en las filiales de empresas multinacionales. En estas condiciones, la gestión del conocimiento ofrece a las empresas colombianas una alternativa para mejorar la productividad y la competitividad.

\section{Abstract}

The problems that organizations face today are related with the dificultéis and failures to carry out processes such as monitoring, following up and control of different activities that companies make to make products and to offer services that respond to the expectations and needs of clients and markets.

Productivity, understood as the effective use of organizational capacities and resources present in the fabrication of goods or service offers, is associated with the knowledge that people get from the actions and activities that are present in diverse processes. This implies the involvement of topics such as knowledge management, total loss control and productivity.

\section{KEY WORDS}

Knowledge management, total loss control, productivity. 


\section{LA EFECTIVIDAD DE LA GESTIÓN EMPRESARIAL}

I

as repuestas creativas e ingeniosas a las dificultades y problemas que enfrentan las organizaciones se logran cuando la gestión del conocimiento se utiliza para «añadir valor, haciendo que su conocimiento sea más eficiente, este mejor conectado y sea tremendamente innovador» ${ }^{1}$.

La efectividad del conocimiento implica organizar la información y el conocimiento para darle una estructura que los haga accesibles en forma ágil para todas la personas y que pueden ser utilizado para agregar valor y responder a las exigencias de los clientes y el mercado. Esto implica desarrollar «la capacidad de una compañía para generar nuevos conocimientos, diseminarlos entre los miembros de la organización y materializarlos en productos, servicios y sistemas $»^{2}$.

La conectividad del conocimiento posibilita asegurar la fluidez y disponibilidad en el momento y lugar donde se requiere para agregar valor a los productos y servicios, que faciliten la generación de respuestas y soluciones para atender a las necesidades de los clientes internos y externos de la organización en forma proactiva. Esta condición implica compartir las mejores prácticas y las respuestas más creativas e ingeniosas a las dificultades enfrentadas.

La innovación en el conocimiento requiere utilizar la percepción y la intuición para responder con ingenio y creatividad mediante la improvisación y experimentación con soluciones diversas, flexibles y alternativas a los desafíos que presenta el escenario competitivo en los tiempos actuales. Las respuestas y soluciones pueden estar expresadas en productos, servicios o procesos.

Para responder a los planteamientos anteriores es necesario generar acciones creativas e ingeniosas que potencien las interacciones de las personas, las organizaciones, las estrategias y los conocimientos, de tal manera que, posibilite el despliegue de todos los talentos de la organización para lograr una posición de vanguardia en los nuevos escenarios competitivos y asegurar un desarrollo sostenible y sustentable.

Las organizaciones que pueden desplegar sus capacidades y recursos con velocidad y flexibilidad y que además gestionan de manera efectiva y productiva la inteligencia, la creatividad y la intuición, desarrollan la habilidad para innovar con rapidez logrando crecimiento y desarrollo en ambiente cambiante con evolución dinámica y permanente.

En estas condiciones la gestión del conocimiento ofrece un gran potencial de desempeño para atender a las exigencias de los nuevos escenarios empresariales que requieren afinar las habilidades y capacidades para gestionar los procesos de percepción, creación de conocimientos y toma de decisiones. La gráfica 1. Gestión del conocimiento muestra estos procesos y los diferentes elementos que los integran.

TISSEN Rene, ANDRIESSEN Daniel, LEKANNE DEPREZ Frank. El valor del conocimiento para aumentar el rendimiento en las empresas. Editorial Pearson educación S.A. Madrid. 2000. P. 37.

2 NONAKA Ikujiro, TAKEUCHI Hirotaka. La organización creadora de conocimiento. Cómo las compañías japonesas crean la dinámica de la innovación. Editorial Oxford University Press. México D.F. 1999. p. 1. 


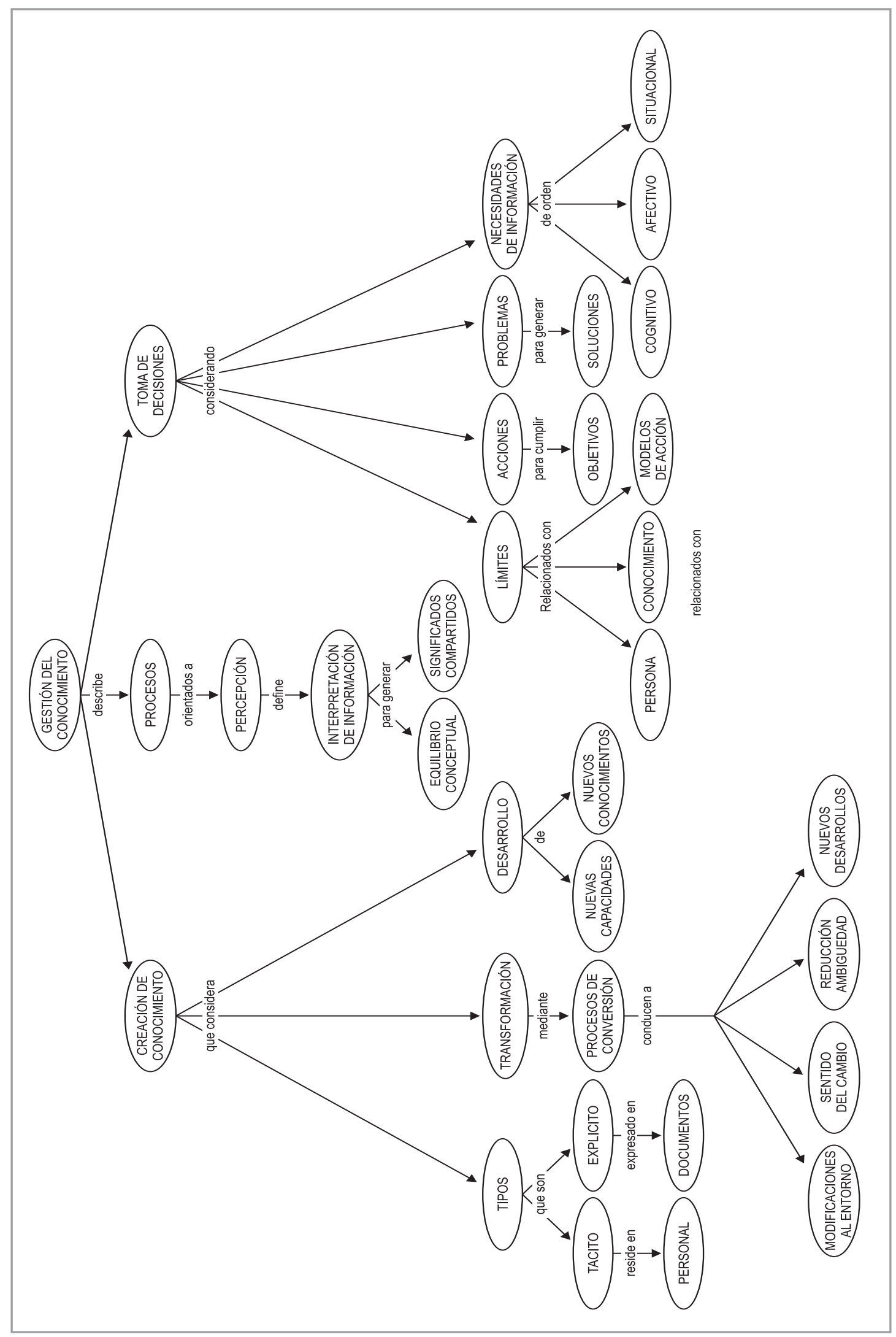


Las organizaciones que gestionan de manera efectiva el conocimiento logran una muy buena «comprensión de los procesos humanos y de la organización a través de los cuales la información se transforma en discernimiento, conocimientos y acción, $\nu^{3}$ para atender de manera imaginativa a los desafíos y dificultades del entorno competitivo de la empresa.

La gestión del conocimiento se aborda desde los procesos que realiza la organización en tres ámbitos esenciales para la sustentabilidad, que corresponden a la percepción, la creación de conocimientos y la toma de decisiones.

Desde el proceso de percepción se enfatiza en los aspectos, elementos y factores que permiten interpretar la información obtenida del entorno empresarial para lograr atender, por una parte, a la búsqueda de un equilibrio dinámico entre flexibilidad y estabilidad de la organización y por otra parte a la generación de significados compartidos por todas la personas involucradas.

La percepción, permite generar orientaciones y directrices para emprender acciones partiendo de las propias concepciones de la realidad empresarial, atendiendo a la ambigüedad en la información y en las acciones de la competencia. Esta condición permite contextualizar el conocimiento mediante procesos de interpretación y traducción de los diferentes sucesos que acontecen en el entorno empresarial.

Desde el proceso de creación de conocimientos desarrollan métodos de conversión de conocimientos tácitos a explícitos y viceversa. El conocimiento tácito corresponde a los saberes, experiencias y percepciones que las personas utilizan para hacer frente a los desafíos y dificultades de su ambiente de actuación, no es evidente y es difícil de expresar por medios formales.

El conocimiento tácito presenta dos dimensiones la «técnica, que incluye las habilidades no formales y muy difíciles de definir que se expresan en el término Know How (saber cómo llevar a cabo una tarea o trabajo).. cognoscitiva. Esta incluye esquemas, modelos mentales, creencias y percepciones tan arraigadas en cada persona que casi siempre las ignoramos» ${ }^{4}$. Solo mediante la creación de los escenarios que promuevan interacciones efectivas y de calidad entre las personas se facilita los procesos de conversión de conocimientos tácitos a explícitos y en sentido contrario.

Para mejorar el desempeño productivo y competitivo de la organización, se requiere enfocar las acciones y actividades de conversión de conocimientos, en el desarrollo de nuevas capacidades y nuevos conocimientos para optimizar y cualificar los procesos y sistemas que permiten la fabricación de productos o la prestación de servicios de excelencia para responder a las cambiantes demandas de clientes consumidores y mercados. Así como, al desarrollo y mejoramiento de los diversos sistemas organizacionales para asegurar la sostenibilidad de la empresa.

Finalmente, la gestión de conocimiento, desde la perspectiva del proceso de toma de decisiones considera cuatro elementos importantes que corresponden a: los límites o limitaciones, las acciones a emprender, los problemas y las necesidades de información en el proceso de toma de decisiones.

\footnotetext{
CHOO Chun Wei. La organización inteligente. El empleo de la información para dar significado, crear conocimiento y tomar decisiones. México: Oxford University Press. 1999. P. 1.

4 NONAKA Ikujiro, TAKEUCHI Hirata. La organización creadora de conocimiento. Cómo las compañías japonesas
} crean la dinámica de la innovación. México. D.F. Editorial Oxford University press. 1999. p. 7. 
Con respecto a las limitaciones del proceso de toma de decisiones, se analiza y evalúa los aspectos relacionados con la persona y las dificultades que enfrenta para manipular grandes volúmenes de información, los hábitos de la mente, los conocimientos y experiencias previas y los modelos mentales entre otros.

En relación al conocimiento es necesario considerar el conocimiento disponible sobre el tema, la dificultad o el problema a enfrentar, el dominio conceptual de las personas, las opciones o alternativas para actuar, la confiabilidad de los conocimientos y demás información existente entre otros aspectos.

El último factor limitante corresponde a los modelos de acción que en algunos casos actúan como catalizadores y en otros como inhibidores del proceso decisional en la organización.

El segundo elemento corresponde a las acciones que en última instancia ponen en marcha una decisión para alcanzar las metas fijadas y cumplir con los objetivos establecidos previamente. Las acciones ponen en evidencia la forma como se actúa y se procede a la ejecución de las diversas tareas y actividades que se realizan en la organización.

Con respecto a los problemas es necesario considerar que en el ámbito empresarial las personas se enfrentan a problemas débilmente estructurados, es decir, que no se dispone de información suficiente para soportar el proceso decisional y adicionalmente, estos problemas tienen múltiples opciones de respuesta; así que, la tarea de resolución de problemas se torna un poco compleja y las personas para hacer frente a esta situación optan por elegir la primera solución que cumpla con los mínimos requisitos exigidos para el problema, en lugar de buscar la mejor a solución o una solución óptima.
Finalmente, con relación a las necesidades de información, se considera que en el proceso de toma de decisiones, existen diversas necesidades de información asociadas a diferentes aspectos o elementos, en este caso, se consideran «las necesidades cognoscitivas, las reacciones afectivas y las dimensiones situacionales $»^{5}$. Las necesidades cognoscitivas están asociadas a los conocimientos, las reacciones afectivas a los sentimientos y emociones de las personas y las dimensiones situacionales corresponden a la situación específica en que realiza el proceso de toma de decisiones.

Desde esta perspectiva, el proceso decisional en las organizaciones necesita integrar información de diversas fuentes y a través de diferentes medios para eliminar o atenuar la incertidumbre y el riesgo en las acciones a emprender. La reducción de la incertidumbre en la toma de decisiones se puede enfrentar por medio de: la delimitación del escenario problémico que requiere la generación de alternativas de solución, el establecimiento de las reglas, preferencias y criterios de elección para atender a los diversos fines e intereses de los involucrados y la exploración de opciones viables y factibles que permitan la generación de diferentes cursos de acción que logren los resultados proyectados para la situación evaluada.

Con relación a las necesidades cognoscitivas el proceso de toma de decisiones requiere acopiar la información para lograr la comprensión de la situación problema, la dificultad o el desafío que debe abordar. Para ello, se propone una estrategia de cuatro elementos que permiten conceptualizar la situación a enfrentar. El primer elemento corresponde a la búsqueda, que esta enfocada en la exploración de las condiciones del ambiente que requieren tomar una decisión o 
exige emprender nuevas acciones. El segundo elemento corresponde a la generación de alternativas de solución con los respectivos planes de acción para resolver la dificultad o iniciar nuevas maniobras. El tercer elemento corresponde a la decisión, es decir, hacer la elección de la mejor o más prometedora solución y la respectiva puesta en práctica. Finalmente, el último elemento es el control que tiene como propósito la verificación de los resultados logrados y su contrastación con los proyectados; en el caso que presenten desviaciones tomar las medidas correctivas pertinentes.

Con respecto a las reacciones afectivas que afectan el proceso de toma de decisiones es necesario tener presente el efecto de los sentimientos, las emociones y las motivaciones de los participantes en el proceso decisional. En este caso se requiere valorar la influencia de los siguientes factores: la complejidad, el grado de estructuración y la incertidumbre en la situación abordada que generan duda y confusión; el riesgo y la dificultad para anticipar los resultados futuros que producen estrés en los participantes; los múltiples intereses de los involucrados que implican diversos tipos de confrontación; $y$ finalmente la distancia entre las aspiraciones personales y las directrices de la organización.

Con respecto a las dimensiones situacionales establece las circunstancias y condiciones en las que se toma la decisión. Por lo general el proceso decisional sucede en dos escenarios diferentes: uno planeado, en el cual las decisiones están debidamente estructuradas con procesos y procedimientos estandarizados y cuentan con los recursos y el soporte para la ejecución. El segundo escenario es eventual, donde el proceso de toma de decisiones presenta situaciones débilmente estructuradas con información incompleta y existen múltiples opciones de respuesta. Estas condiciones novedosas requieren de la utilización del juicio, la intuición y la creatividad para el abordaje de la situación, aplicando la experiencia, la heurística, las percepciones de quién toma la decisión, que despliega sus propias tácticas y estrategias para la generación de alternativas de solución.

\section{GESTIÓN DEL CONOCIMIENTO Y APRENDIZAJE}

E n la actualidad las organizaciones requieren atender en forma efectiva y con gran velocidad a los desafíos que se generan en el ambiente en el cual desarrollan las diferentes actividades. Esto exige personas altamente capacitadas y con la habilidad para decidir que hacer y como actuar frente a una situación determinada, un cliente molesto, un proveedor que no ha cumplido ó al que no se le ha pagado.

Una forma de generar respuestas efectivas a estos desafíos es preparar y ayudar a las personas para que desarrollen las competencias necesarias que aseguren un buen desempeño personal y profesional. Desde esta perspectiva una opción, es partir de la concepción de gestión del conocimiento para garantizar el desarrollo de competencias en las organizaciones.

En las actuales condiciones de complejidad y cambio permanente; las empresas, los empresarios, las personas y la sociedad en general requieren generar valor agregado, es decir, deben aprender a crear, utilizar y gestionar en forma 
efectiva y productiva el conocimiento para lograr un buen desempeño competitivo y poder ubicarse en un escenario global dinámico y altamente competitivo para las personas, las organizaciones y la sociedad en general.

Agregar valor en forma creativa e innovadora requiere de alta flexibilidad de pensamiento y la capacidad de acción proactiva para anticiparse a las necesidades, deseos y expectativas de los clientes que son cada vez más exigentes, están mejor informados y tienen más poder de decisión.

El aprendizaje se convierte en un factor determinante en la productividad de las organizaciones porque permite ejecutar las acciones y actividades de una manera diferente y considerando alternativas y opciones. Desarrolla la capacidad para resolver problemas con flexibilidad, agilidad y efectividad logrando un desempeño de excelencia, es decir, se vuelve competente para responder a las demandas de los consumidores y el mercado.

Por consiguiente, aprendizaje implica desarrollo de competencias para integrar nuevos conocimientos, generar modelos de acción alternativos, ampliar la habilidad para realizar interacciones con versatilidad y calidad, con el fin de hallar respuestas imaginativas y creativas a las expectativas y necesidades cambiantes de los clientes.

\section{LAS INTERACCIONES EN LA GESTIÓN DEL CONOCIMIENTO}

E

conocimiento en las organizaciones por lo general es de tipo tácito y se encuentra en los cerebros de las personas, por lo tanto, se requiere hacerlo disponible para que toda la organización se pueda beneficiar de él. Esto exige de un adecuado proceso de interacción entre los diferentes elementos componentes de un sistema de conocimiento en la organización. En la Gráfica 2. Las interacciones en la gestión del conocimiento, o que se explican en los párrafos siguientes se muestra una de las posibles formas de interacción.

Los sistemas de gestión del conocimiento son los que movilizan y ponen en acción el conocimiento en las organizaciones y por lo general corresponden a: el conocimiento cognitivo, las competencias de las personas, la creatividad autorregulada y las interacciones que posibilitan integrar los diferentes elementos del sistema para la generación de soluciones efectivas a los problemas y dificultades que plantea un entorno empresarial dinámico y altamente competitivo.

- El conocimiento cognitivo corresponde a los dominios conceptuales que poseen las personas en un campo específico del saber, lo que posibilita identificar las estructuras y procesos para desarrollar síntesis a partir del análisis, lo que permite la generación de proposiciones, para ejecutar procedimientos y procesos de manera efectiva y productiva en el mundo empresarial real. 
Gráfico 2

Interacciones en la gestión del concimiento

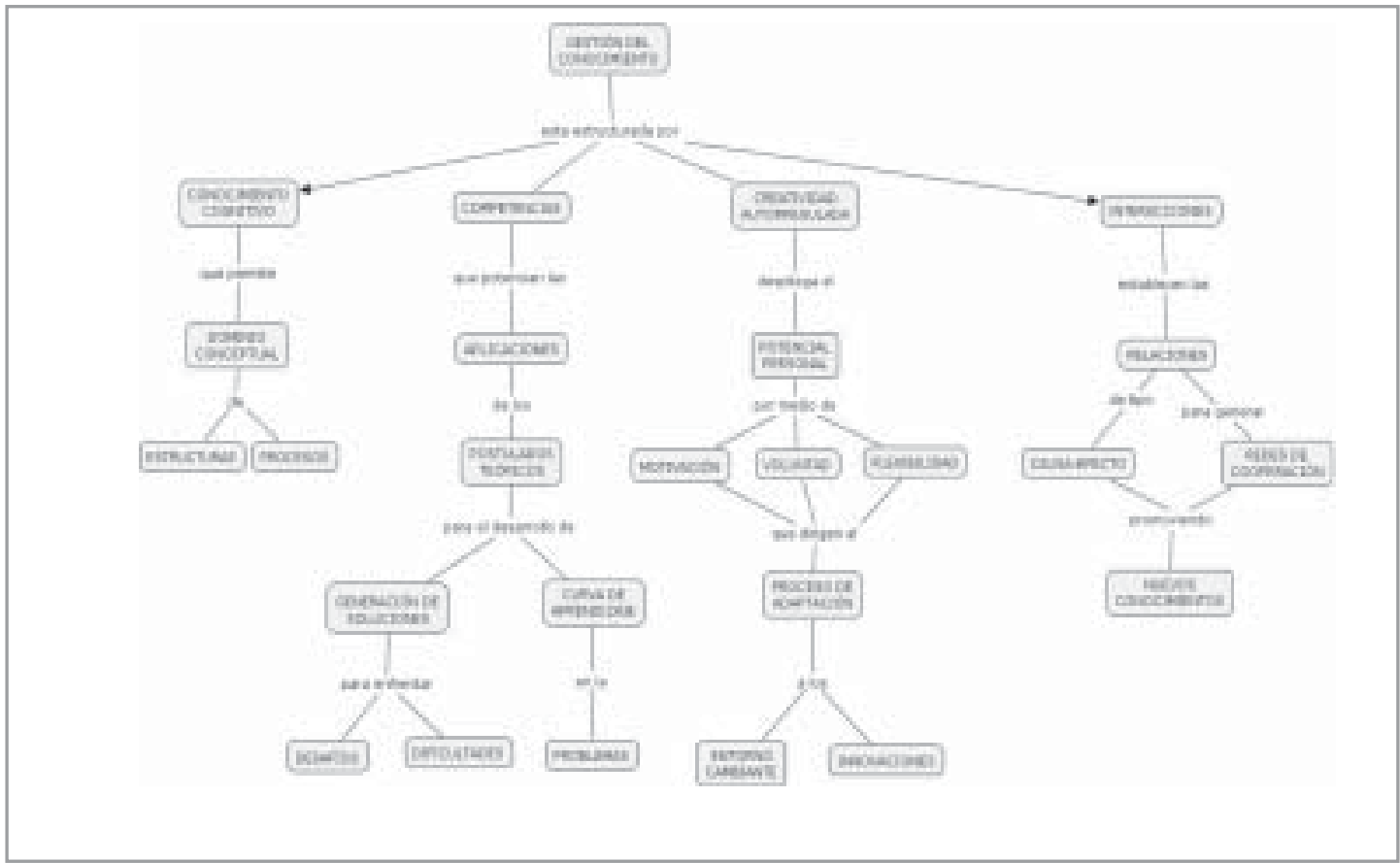

Los sistemas de gestión del conocimiento son los que movilizan y ponen en acción el conocimiento en las organizaciones y por lo general corresponden a: conocimiento cognitivo, competencias de las personas, creatividad autorregulada e interacciones que posibilitan integrar los diferentes elementos del sistema para la generación de soluciones efectivas a los problemas y dificultades que plantea un entorno empresarial dinámico y altamente competitivo.

- El conocimiento cognitivo corresponde a los dominios conceptuales que poseen las personas en un campo específico del saber, lo que posibilita identificar las estructuras y procesos para desarrollar síntesis a partir del análisis, lo que permite la generación de proposiciones, para ejecutar procedimientos y procesos de manera efectiva y productiva en el mundo empresarial real.
- Las competencias concebidas como el potencial de aplicación de los principios y postulados teóricos de un campo particular del saber, a la generación de soluciones prácticas, a las dificultades y desafíos que presenta el entorno empresarial. La competencia permite el desarrollo de la curva de aprendizaje de las personas en una forma adaptable y flexible para resolver los problemas complejos del mundo empresarial real.

- La creatividad autorregulada permite el despliegue de todo el potencial de la persona poniendo en acción la voluntad, la motivación y la capacidad de adaptación para ejecutar procesos creativos de manera consciente que permite adaptarse a las cambiantes condiciones del entorno y a las innovaciones que ponen en riesgo de obsolescencia las competencias actuales de las organizaciones y de sus miembros. 
- Finalmente, las interacciones se convierten en el elemento de cohesión que potencia la relación de los diversos componentes del sistema de gestión del conocimiento en la organización. Son las que determinan las relaciones causa-efecto y generan las redes de cooperación y colaboración para la generación de nuevos conocimientos.

\section{LA EVOLUCIÓN DE LA GESTIÓN DEL CONOCIMIENTO}

La

a conceptualización de la gestión del conocimiento presentó un fuerte desarrollo en la década de los noventa, época en la cual surge una serie de modelos que explican desde diversas perspectivas y concepciones la gestión del conocimiento en las organizaciones. Entre estos modelos se pueden mencionar los siguientes:

- Pilares de la gestión del conocimiento. El modelo que conceptualiza la gestión del conocimiento como un conjunto de funciones para la creación, demostración, aplicación y distribución del conocimiento fue propuesto por Wiig a principios de la década de los 90 y establecía que la gestión del conocimiento se estructuraba alrededor de tres pilares: el primero relacionado con la exploración y adecuación del conocimiento, el segundo referido a la evaluación del valor del conocimiento y finalmente, el tercer pilar hace referencia a los procesos de gestión del conocimiento.

- Conversión del conocimiento. Este modelo fue propuesto por Nonaka y Takeuchi y parte de la concepción de que existen dos tipos de conocimiento: tácito y explícito, que mediante diversos procesos puede lograr la modificación de los conocimientos tácitos para convertirlos en explícitos y luego el conocimiento explícito transformarlo en tácito. Estos procesos son: socialización que permite la conversión del conocimiento tácito individual en tácito colectivo mediante el intercambio de ideas, la interacción entre las personas, compartir experiencias y tradiciones, narración de las interpretaciones y aplicaciones personales de los documentos y manuales de la organización, entre otras. Exteriorización que por medio de interacciones este proceso convierte el conocimiento tácito en conocimiento explícito, por lo general utiliza metáforas y analogías para manifestar los conceptos. Combinación que posibilita la conversión de conocimiento explícito individual a conocimiento explícito organizacional, se logra por medio de la reunión del conocimiento obtenido de diferentes fuentes; esto implica realizar procesos de categorización y clasificación del conocimiento incorporado. Interiorización a partir de la apropiación y asimilación del conocimiento explícito organizacional se convierte en conocimiento tácito individual.

- Capacidades y creación de conocimiento. El modelo fue planteado por Leonard-Barton hacia mediados de la década del noventa. Éste se estructura alrededor de dos elementos fundamentales: las capacidades básicas organizacionales y las actividades de creación de conocimientos. 
Las capacidades básicas organizacionales corresponden a: los sistemas físicos que definen la curva de aprendizaje o sea las competencias acumuladas y que se han hecho explícitas en la organización; los sistemas de gestión relacionados con las actividades de acumulación y distribución del conocimiento, y la generación de mecanismos y vías de acceso a estos conocimientos. Las competencias de los trabajadores de la organización referidos a las actitudes y conocimientos que establecen los mecanismos para la producción de conocimientos en la organización. La última capacidad corresponde a los valores y normas de la organización que establecen las formas y tipos de conocimiento requerido y los mecanismos de control del conocimiento.

Con respecto a las actividades de creación de conocimiento el modelo establece las siguientes: resolución creativa de problemas orientada a la producción de conocimiento mediante la fabricación de productos y la prestación de servicios; importación de conocimiento referida a la adquisición del conocimiento generado por otros; implanta-ción orientado a la aplicación del conocimiento por medio de la integración de metodologías, tecnologías y demás instrumentos y herramientas; finalmente, la experimentación orientada al desarrollo de capacidades y competencias útiles en el futuro.

- Organización del conocimiento. El modelo fue desarrollado por Arhtur Andersen y APQC, está integrado por el conocimiento organizativo, los procesos de gestión del conocimiento que se estructura como un ciclo y los instrumentos de la gestión del conocimiento. Los procesos de gestión del conocimiento interactúan de manera integrada e integral para lograr un mejor desempeño y corresponden a: intercambio, creación, identificación, recopilación, adaptación, organización y aplicación. Además el modelo es afectado por los instrumentos que optimizan y hacen efectiva la gestión del conocimiento; estos son: liderazgo, cultura organizacional, tecnología y valoración.

- Organización inteligente. El modelo planteado por Chun Wei Choo establece que las organizaciones en el desarrollo de sus actividades realizan tres procesos para gestionar el conocimiento: interpretación orientada a la comprensión de la información para convertirla en conocimiento útil y aplicable en la organización; creación de conocimiento por medio de la generación de respuestas efectivas y creativas a las dificultades y desafíos que propone el ambiente de actuación $\mathrm{y}$ toma de decisiones que determina la forma como la organización procesa la información y el conocimiento para enfrentar la incertidumbre en sus acciones y actuaciones.

- Capital intelectual. Entendido como los «activos identificables de naturaleza no monetaria y sin sustancia física destinados a ser usados en la producción o suministro de bienes y servicios. $\aleph^{5}$ El modelo fue desarrollado por Leif Edvinsson, Hubert Saint Onge, Patrick Sullivan y Gordon Petrash enfocado hacia la organización de los recursos del conocimiento estructurado alrededor de tres elementos: capital humano referido al conocimiento, competencias, capacidades, habilidades y experiencias que poseen y generan las personas dentro

5 Edvinsson Leif, Malone Michael S. El capital intelectual. Barcelona: Gestión 2000,1999. 
de la organización; capital estructural entendido como los conocimientos que se han ordenado, clasificado y acumulado en los procesos, la estructura, los sistemas tecnológicos y administrativos, los modelos, los sistemas de información y la cultura de la organización y el capital relacional que se obtiene de la interacción con los proveedores, clientes, competidores y demás agentes del entorno empresarial.

- Transferencia de conocimiento. El modelo fue presentado por Szulanski como instrumento de análisis de la estructura interna de la organización con el propósito de realizar la transferencia de las mejores prácticas organizacionales.

El modelo está compuesto de dos elementos: las fases de la transferencia que corresponden a inicio que reconoce y satisface las necesidades de conocimiento; implantación que dirige y promueve la transferencia de conocimiento, aplicación que utiliza el conocimiento transferido e integración que asegura la apropiación e interiorización del conocimiento. Los factores que afectan la transferencia del conocimiento, están integrados por las características de la transferencia, las características de la fuente, las características del destinatario y las características del contexto.

- Proceso de gestión. Fue desarrollado por KPMG Peat Marwick para los procesos de consultoría y esta enfocado a la creación, apoyo e intercambio de los saberes, capacidades y habilidades de las personas de la organización para agregar valor que asegure la satisfacción de los clientes de la organización.
El modelo está integrado por seis elementos que son: adquisición, orientado a la creación de conocimientos y desarrollo de contenidos; indexación, que tiene como propósito la organización, clasificación y categorización del conocimiento; filtración, entendido como proceso de depuración y estructuración del conocimiento; enlace dirigido a la identificación de relaciones, conexiones, e interacciones, distribución, orientado a la agrupación y entrega del conocimiento por medio de diversas tecnologías y la aplicación que corresponde a los diferentes usos del conocimiento en la organización.

- Activos intangibles. Este modelo desarrollado por Sveiby define la naturaleza del conocimiento organizacional como activo intangible y se estructura alrededor de tres componentes: estructuras externas corresponde al conocimiento generado por los procesos de interacción con el entorno, estructuras internas define el conocimiento generado mediante interrelaciones al interior de la organización y las competencias del trabajador referidas a las actitudes y conocimientos de los miembros de la organización.

- Etapas de Gestión. El modelo fue planteado por Van der Spek y Spijkervet definiendo la gestión del conocimiento como un ciclo que comprende cuatro actividades que se desarrollan de manera secuencial. «Estas actividades son: revisión, conceptualización, reflexión y acción ${ }^{7}$ y se ejecutan en un ambiente donde interactúan personas, tecnologías, información y cultura organizacional. La revisión esta referida a la búsqueda, clasificación y modelado del conocimiento existente, la conceptualización relacionada

\footnotetext{
7 WIIG Karl, DE HOOG Robert, VAN DER SPEK Robert, Supporting knowledge management: a selection of methods and techniques. En http://hcs.science.uva.nl/kennisenleren/cursus0102/wiig2.doc Consultado febrero 6 de 2007.
} 
con la comprensión de las fortalezas y debilidades del conocimiento disponible en la organización, la reflexión orientada a la evaluación y análisis del conocimiento existente para determinar los conocimientos requeridos y planear los procesos de apropiación e integración de nuevos conocimientos, finalmente la actuación enfocada a la optimización del conocimiento existente para generar nuevos conocimientos, distribuirlos y combinarlos para asegurar la utilización en la satisfacción de las necesidades de clientes y consumidores, mediante la comparación, evaluación y contrastación de los resultados obtenidos frente a los esperados en el entorno empresarial.

\section{GESTIÓN DEL CONOCIMIENTO Y PRODUCTIVIDAD UNA PROPUESTA}

\section{L}

a productividad en las organizaciones está determinada por la forma en que se optimiza y racionaliza la aplicación de los limitados recursos disponibles en la elaboración de productos o la prestación de servicios. En esencia esto es la habilidad para gestionar los recursos; sin embargo en un entorno dónde todos compiten por los limitados recursos, es necesario lograr un uso efectivo de los mismos para lograr mejores resultados.

La efectividad en la utilización de los recursos y capacidades de la organización requiere «ejercer un liderazgo efectivo en seguridad y otorgar una protección responsable a las personas, a los equipos, a los materiales y al ambiente. $\|^{8}$ Esto implica enfocar los esfuerzos de la gestión empresarial en una acción integradora que considere la seguridad, la calidad, la productividad de los procesos productivos y el control de costos.

Una de las formas más empleadas en estos casos corresponde al establecimiento de programas, normas de actuación y estándares de desempeño que dirijan las interacciones de los diferentes factores del sistema productivo. Por lo general, es el más utilizado y que puede convertirse en el más efectivo sistema de control de riesgos, es cuando se trabaja sobre las causas del riesgo y no sobre los síntomas, que es una tendencia generalizada en las organizaciones; esto equivale a tapar una gotera sin hacer la revisión del techo.

Por lo general, las organizaciones son muy buenas en el proceso de planeación y en materia de prevención de riesgos ocurre igual, se planea muy bien las acciones y actividades a realizar, incluso se diseñan excelentes programas para el control de riesgos y se ponen en marcha sin establecer los mecanismos de control que permitan la verificación de los resultados esperados en cada caso.

Aunque parece evidente aún no es clara la relación entre control de riesgos y la productividad. Para lograr precisión al respecto

8 BIRD Frank E. Jr, GERMAIN George L. Liderazgo práctico en el control de pérdidas. La conservación de gente, propiedad, proceso y ganancias. Editado Det Norske Veritas (USA), Inc. Atlanta. Georgia. 1990. P. 3. 
a continuación se define esta relación. Las organizaciones tienen una mayor productividad cuando controlan los riesgos que generan pérdidas en el sistema productivo y evitan que estas pérdidas se materialicen. Esto se logra utilizando sistemas efectivos de control de riesgos.

La relación entre control de riesgos y productividad es clara, sin embargo, no hay claridad sobre cómo se relaciona con la gestión del conocimiento, esto es un poco más complejo y tiene que ver con la comprensión de los diferentes elementos que integran el sistema productivo y la forma como se relacionan e interactúan para la generación de los productos y servicios en la organización.

Para lograr comprender cualquier sistema es necesario conocerlo, identificar sus funciones, los elementos que lo constituyen, la forma como se interrelacionan estos elementos y los riesgos potenciales de la utilización e interacción de estos elementos. Esto es en esencia conocimiento, que requiere ser gestionado para obtener los resultados esperados del sistema en forma efectiva y productiva. Para comprender mejor esta relación se presenta el Gráfico 3. Productividad y gestión del conocimiento que se describe y explica en los párrafos siguientes.

Gráfico 3

Productividad y gestión del conocimiento

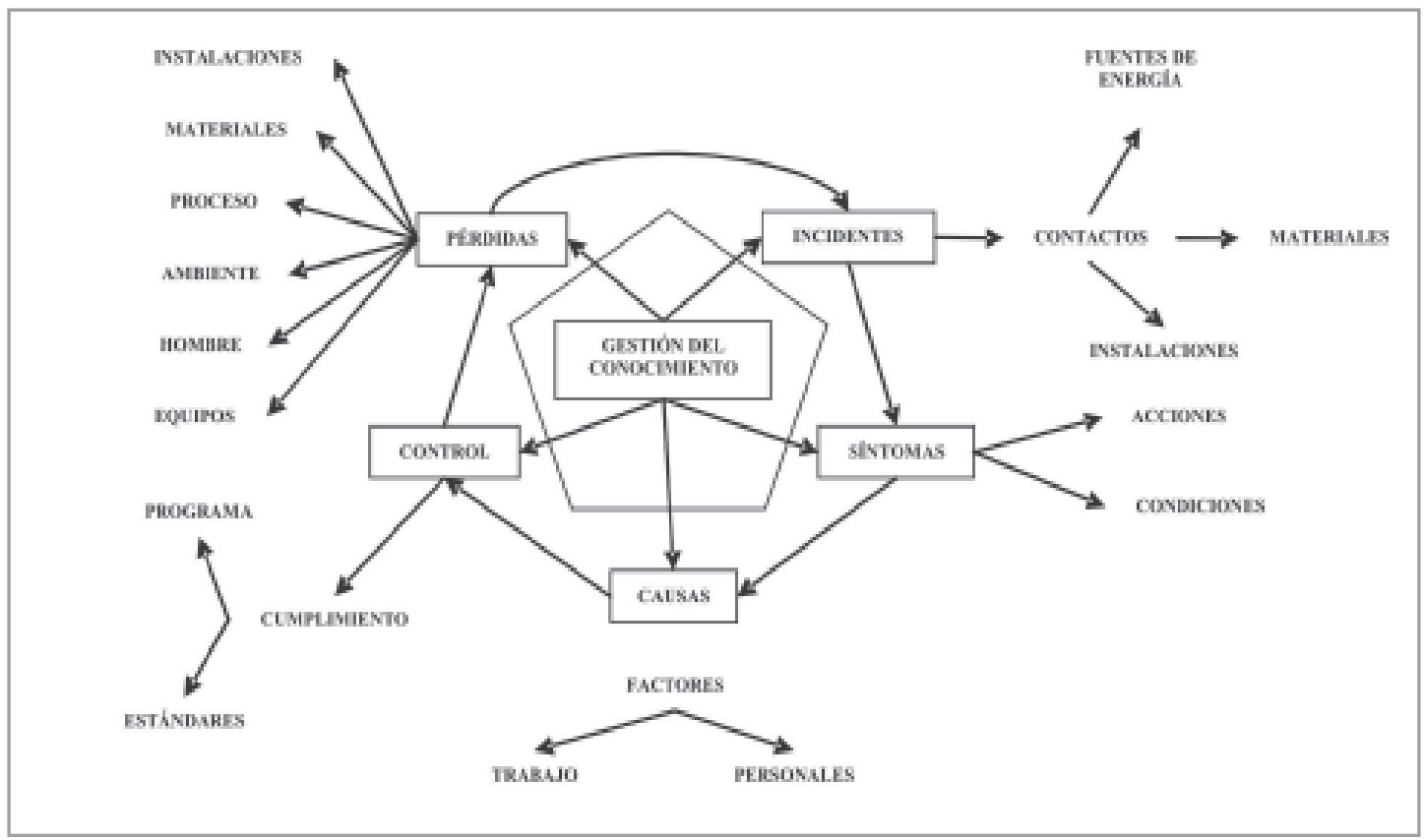

Desde esta concepción, la gestión del conocimiento parte de una evaluación y análisis de lo peor que podría suceder en la organización, es decir, las pérdidas potenciales que se puede presentar en el desarrollo de las actividades cotidianas y su efecto en el sistema productivo, para hacer una valoración del impacto de cada suceso en la empresa. A partir de la calificación de las pérdidas potenciales se establecen los mecanismos de control que permitan actuar sobre las causas de cada uno de los riesgos que afecta a la organización. 
Las organizaciones necesitan desarrollar estrategias y procesos de gestión que potencien el uso de los recursos y se elimine o al menos se atenúe el impacto de los diversos riesgos, que en términos generales son los generadores de las pérdidas en el entorno empresarial. Estas pérdidas están asociadas a los factores que integran el sistema productivo de las organizaciones.

Los factores sobre los que se evidencian las pérdidas son los siguientes: materiales, equipos, procesos, instalaciones, el hombre y el ambiente. Cada factor involucrado presenta sus propios niveles de riesgo en la operación y funcionamiento de la organización, lo ideal en cualquier proceso de control de riesgos, es eliminar o aislar el factor generador del riesgo. En este caso la decisión no es sencilla, porque para lograr la productividad se requiere que todos los factores trabajen de manera integrada e interrelacionada.

Si se eliminan los materiales del sistema productivo, se genera carencia de materias primas para la fabricación de los productos o la prestación de los servicios; el aislamiento de este factor hace lento o impide el proceso de transformación que es lo que genera los productos en la organización. Igual ocurre con el aislamiento de los demás factores del sistema productivo.

Entonces, ¿cuál es la solución? Al respecto, los expertos proponen seleccionar factores seguros para el proceso productivo, que en la mayoría de veces resulta poco factible o simplemente no es práctico y en algunos casos es imposible de realizar, porque el riesgo está asociado al proceso, a los materiales o las instalaciones, entre otros.

Desde esta perspectiva, lo mejor es realizar procesos de gestión que aseguren un manejo adecuado de los factores para atenuar el riesgo y el impacto de su efecto cuando se presentan incidentes en el sistema productivo. Es así como, la respuesta requiere de mayor precisión porque es necesario establecer qué se debe gestionar y cómo hacerlo para lograr una mayor efectividad en las decisiones a tomar y las acciones a emprender para mejorar la seguridad del sistema productivo.

Los incidentes corresponden a la expresión más frecuente de la existencia de fallas en los sistemas de la organización y que ponen en evidencia las pérdidas potenciales a que está expuesta la empresa. Los incidentes son manifestaciones de las interacciones inadecuadas entre los elementos del sistema, generando contactos con fuentes de energía, instalaciones y materiales que pueden generar pérdidas, lesiones o daños graves en el sistema, en los factores y en la organización. Los incidentes ponen en evidencia los aspectos visibles de la situación, que por lo general corresponde a los síntomas del problema o las dificultades que es necesario atender para eliminar, evitar o prevenir el riesgo y la pérdida asociada.

Los síntomas que son las evidencias que presentan las desviaciones en los sistemas y los procesos, muestran la existencia de deficiencias en la organización, están asociadas a las acciones de las personas y las condiciones del ambiente que generan los riesgos y las exposiciones de la organización a pérdidas significativas en sus procesos, productos, instalaciones y materiales entre otros.

Una evaluación y análisis en profundidad de los síntomas ponen en evidencia las verdaderas causas que se esconden detrás de los síntomas. La verificación detallada de cada incidente permite establecer los factores que determinan la exposición a pérdida. Estos factores están asociados a la identificación de 
las razones de las actuaciones de las personas, factores personales, que generan riesgo y el segundo factor corresponde a la forma como se realizan las actividades, llamados factores del trabajo y que exponen la organización a pérdidas.

Para eliminar, evitar o prevenir las pérdidas en las organizaciones es necesario realizar procesos de gestión efectivos, que planeen, organicen dirijan y controlen las acciones y actividades que se realizan en la organización para la fabricación de los productos y la prestación de servicios. Por lo general, las organizaciones realizan sólo las tres primeras funciones y desatienden la función de control, que permite la verificación de los resultados logrados frente a los proyectados, identificando las desviaciones y las respectivas causas.

Una vez identificadas las causas es conveniente explorar los diferentes síntomas que evidencia cada una de ellas y qué tipos de incidentes genera en el sistema productivo de la organización. De la misma manera, los posibles daños o efectos sobre los factores del sistema productivo.

En este caso se puede utilizar una estrategia de solución de problemas, como los diagramas causa-efecto, que permiten identificar el problema, es decir, la situación o la condición no deseada; luego mediante un proceso de recolección de información definir, la que básicamente hace referencia a establecer el qué o efecto no deseado, y el quién o sujeto involucrado.

Una vez definido el problema se procede al diagnóstico, es decir, descubrir las causas que afectan al sujeto y producen el efecto. Finalmente, con este cuadro clínico se procede a generar diversas opciones de solución que permitan actuar sobre las causas para eliminar o atenuar el impacto del efecto sobre el sistema productivo y la organización. El Gráfico 4. Solución de problemas y gestión del conocimiento, que presenta a continuación describe esta situación.

Gráfico 3

Productividad y gestión del conocimiento

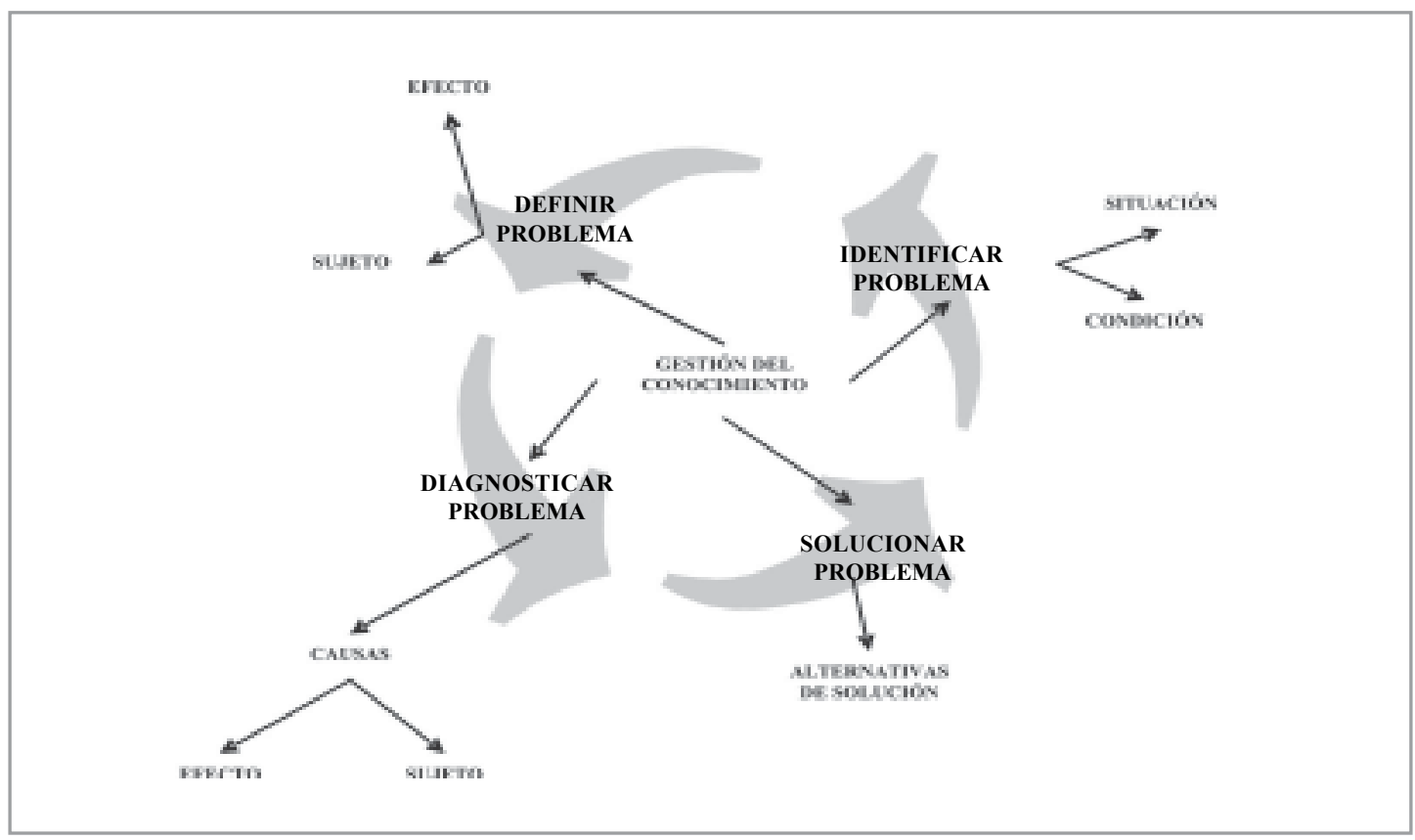


La identificación del problema está asociado a dos elementos; la situación que establece lo que sucede en la organización, es decir cuál es el evento o fenómeno que genera la dificultad o causa el problema y las condiciones que corresponden a las circunstancias sobre la forma como se presentan los acontecimientos en la situación particular que se analiza.

Una vez identificado el problema, es decir, determinadas las circunstancias que han causado el evento es necesario definir el problema, que en esencia requiere establecer los efectos y el sujeto. Los efectos están referidos a las consecuencias o sea qué resultados han producido sobre el sistema o los diferentes elementos constitutivos. El sujeto hace referencia a los actores involucrados, es decir, quienes han participado o participan en la situación o el problema que enfrenta la organización.

Una vez definido el problema es necesario realizar el diagnóstico que pretende establecer las causas que afectan al sujeto y generan el efecto o la desviación de los resultados esperados. El propósito del diagnóstico es determinar las causas del evento y su influencia en la ocurrencia del mismo, es decir, los efectos sobre la organización y el funcionamiento y operación de sus sistemas y procesos. La definición de las causas que influyen el sujeto pretende estimar el impacto sobre los actores en la situación concreta y particular analizada.

Finalmente, diagnosticado el problema e identificadas las causas que producen el efecto y que influyen en los actores, se procede a la generación de soluciones que eliminen o atenúen el impacto de las causas sobre los actores y se controle el efecto generado por el problema, corrigiendo las desviaciones en los procesos y sistemas de la organización.

En síntesis, un adecuado sistema de verificación y control permite mantener bajo control los efectos negativos sobre la organización y las consecuencias que afectan a los actores, mejorando el desempeño de la organización y por lo tanto su productividad. Esto sólo es posible cuando se tiene una muy buena comprensión de la forma como operan y funcionan los sistemas y proceso en la organización, lo cual se puede hacer mediante la aplicación de la gestión del conocimiento. 


\section{Conclusiones}

Comprender el conocimiento que subyace en la organización permite hacer frente de una manera efectiva a los desafíos de entorno empresarial dinámico y altamente competitivo y crear opciones que aseguren la sostenibilidad.

La complejidad generada por la globalización agrega nuevos elementos a la situación y expone las empresas a nuevos retos. Esto afecta la productividad, es decir, la habilidad de la organización para utilizar sus capacidades y recursos de una manera efectiva. Sin embargo, la aplicación de los principios y postulados de la gestión del conocimiento permite hacer frente a estas dificultades.

La investigación sobre el desarrollo y las aplicaciones de la gestión del conocimiento en diferentes organizaciones evidencia los beneficios potenciales de su utilización como herramienta para el mejoramiento de la productividad y la competitividad en las organizaciones.

La aplicación del modelo propuesto permite a las organizaciones mejorar la productividad porque optimiza la utilización de los recursos y capacidades disponibles, al tiempo que contribuye a un desempeño competitivo más efectivo.

Finalmente, una efectiva gestión del conocimiento permite identificar, formular y resolver problemas, generando alternativas de solución desde la comprensión de las capacidades y recursos de la organización y las posibilidades y limitaciones del entorno empresarial. 


\section{Bibliografía}

ARGYRIS Chris. Sobre el aprendizaje Organizacional. Segunda edición. Oxford University Press. México D.F. 2001. 696 p.

BIRD Frank E. Jr. Liderazgo práctico en el control de pérdidas. La conservación de gente, propiedad, proceso y ganancias. Editado Det Norske Veritas. (USA), Inc. Atlanta Georgia. 1990. $442 \mathrm{p}$.

CHOO Chun Wei. La organización inteligente. El empleo de la información para dar significado, crear conocimiento y tomar decisiones. México: Oxford University Press. 1999. 346 p.

EDVINSSON Leif; MALONE Michael S. El capital intelectual. Barcelona: Gestión 2000, 1999.

GALLEGO Domingo J. ONGALLO Carlos. Conocimiento y gestión. Editorial Pearson Educación. S.A. Madrid. 2004. 193 p.

HARVARD BUSINESS REVIEW Gestión del conocimiento. Harvard Business Review. Editorial Deusto. Bilbao. 2000. 245 p.

KROGH Georg von. ICHIJO Kazuo. NONAKA Ikujiro. Facilitar la creación de conocimiento. Como desentrañar el misterio del conocimiento tácito y liberar el poder de la innovación. Editorial Oxford University Press. México. 2001. 469 p.

LEONARD B. Dorothy. Wellsprings of knowledge. Building and sustaining the sources of innovation. Editorial Harvard Business School Press. Boston Massachusetts. 1995. 334 p.

NONAKA Ikujiro. TAKEUCHI Hirotaka. La organización creadora de conocimiento. Como las compañías japonesas crean la dinámica de la innovación. México: Oxford University Press. S.A. 1999.

SUMANTH David J. Ingeniería y administración de la productividad. Editorial Mc Graw Hill. México D.F. 1993. 547 p.

TISSEN Rene, ANDRIESSEN Daniel, LEKANNE DEPREZ Frank. El valor del conocimiento para aumentar el rendimiento en las empresas. Editorial Pearson educación S.A. Madrid. 2000. 306

WIIG Karl, DE HOOG Robert, VAN DER SPEK Robert, Supporting knowledge management: a selection of methods and techniques. En http://hcs.science.uva.nl/kennisenleren/cursus0102/ wiig2.doc 


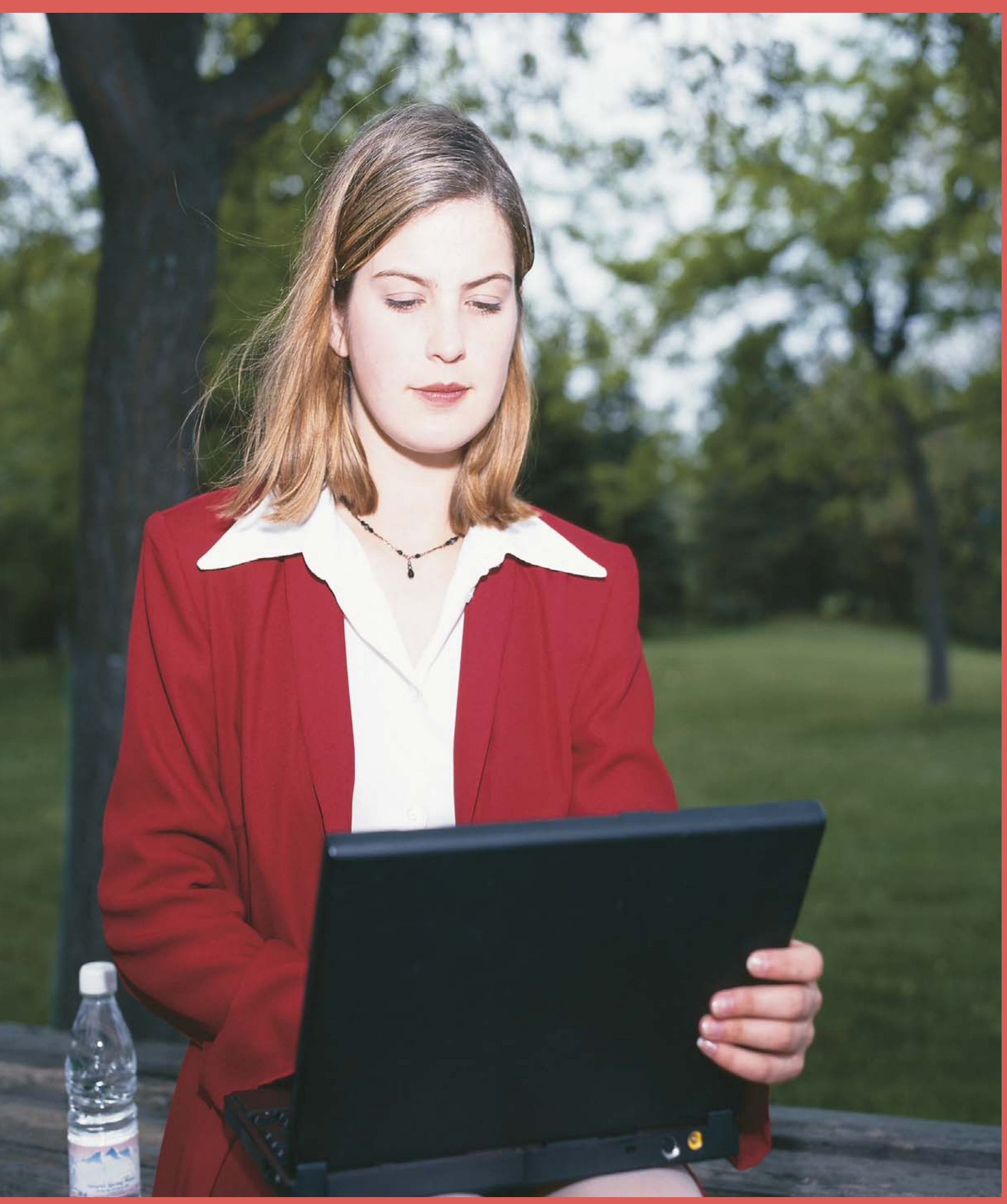

